\title{
Les Hyménoptères et les conditions hygrométriques
}

Jean Leclercq

Citer ce document / Cite this document :

Leclercq Jean. Les Hyménoptères et les conditions hygrométriques. In: Bulletin de la Société entomologique de France, volume 51 (3), mars 1946. pp. 44-48;

https://www.persee.fr/doc/bsef_0037-928x_1946_num_51_3_15875

Fichier pdf généré le 03/07/2019 
vent pas être considérés comme définitifs. Toutefois, nous pouvons en déduire les conclusions préliminaires suivantes :

10 Les pousses de certaines variétés de pommes de terre disposent de moyens d'échapper à la destruction complète par les Insectes printaniers du Doryphore. $2^{\circ}$ Cette résistance partielle est due tantôt à une moindre affinité (Marszalek, Wohltmann), tantôt au développement plus vigoureux a'une variété appréciée par le Doryphore (Sobieszynskie, Rosafolia).

30 Les variétés tardives et dégénérées succombent le plus vite.

\section{Station de Zoologie agricole du Sud-Ouest.}

\section{Les Hyménoptères et les conditions hygrométriques}

$$
\text { par Jean LeclercQ }
$$

Cet article n'a d'autre but que de montrer à l'aide de documents bibliographiques et de quelques résult ats d'expériences personnelles, comment certains Hyménoptères réagissent aux conditions hygrométriques. Il n'a aucunement la prétention d'exposer de façon complète les relations variées qui peuvent exister entre les Hyménoptères, leur bionomie et leur activité, et l'humidité atmosphérique. L'expérimentation en ce domaine est encore trop peu avancée, les principaux types d'Hyménoptères ne lui ont pas encore tous été soumis et, comme on pourra le voir, il n'existe pas encore un seul Hyménoptère dont on connaisse la réaction à l'humidité aı cours du développement complet, depuis l'œuf jusqu'à l'adulte.

\section{Hyménoptères Symphytes \\ (phytophages)}

Alfred Giard montra dès 1894 que les larves de Selandria salina Giard (Tenthrédinide) peuvent vivre plusieurs mois en " anhydrobiose ", c'est-à-dire en un état de diapause où l'animal reste immobile et attend passivement l'humidification du milieu pour continuer son développement.

Lophyrus pini L. (Lophyride) est le seul symphyte qui ait été étudie avec quelque attention. D'après K. Gorswaj.p (1935), ses fausses chenilles sont faiblement " euryhygres" (1), défavorisées légèrement par la sat uration hygrométrique et fortement par la sécheresse extrême. Le même auteur rapporte, en 1941, qu'il a en vain essayé de mettre en évidence un hygrotropisme positif ou négatif chez les mêmes fausses chenilles.

\section{Hyménoptères Térébrants \\ (généralement entomobies, quelques groupes phytophages)}

Les larves de l'Eurytoma amygdali End., Chalcidide phytophage vivant dans les amandes, ont été étudiées expérimentalement par R. Ashber (1932). A la température de $10^{\circ} \mathrm{G}$., ces larves en diapause hivernale, supportent toutes les conditions

(1) J'appelle euryhygres (francisation du euryhygr de G. Geistrandr, 1937), les formes tolèrant sans dommages une grande étendue de l'échelle hygrométrique (zone optimale étendue). Je nomme, par contre, sténohygres (francisation du stenohygr de G. GEisthanDT, 1937) les formes exigeant pour survivre et se développer une zone étroite du gradient hygrométrique (én général, le voisinage de la saturation). 
hygrométriques échelonnées entre 10 et $100 \%$ d'humidité relative ; elles donnent des nymphes lorsqu'elles sont maintenurs tout l'hiver dans ces conditions. Par contre, à $15^{\circ} \mathrm{C}$., ne réussissent à nymphoser que les larves qui ont vécu leur hibernation entre 40 et $90 \%$ d'humidité relative.

G. C. Ullyetr (1936) a étudié la réaction à l'humidité des différents stades du Microplectron fuscipennis, Chalcidide parasite das Lophyres. Ses stades de croissance sont pratiquement indépendants de l'humidité, ce que l'auteur considère comme le résultat de la protection du cocon de l'hôte. Les adultes vivent et pondent d'autant plus longtemps que l'air est plus humide, la longévité et la durée de la ponte étant proportionnelles à la déficience de saturation. Tous les stades sont nettement euryhygres.

H. O. Lund (1934) a expérimenté sur le Trichogramma minutum Riley (Chalcidide) qui parasite les oufs du Sitotroga cerealella Oliv.(Microlep.). Ses stades de croissance sont euryhygres mais ils donnent des adultes en plus grand nombre et plus rapidement quand les élevages sont effectués en atmosphère saturée.

On n'a pas manqué d'étudier les effets de l'humidité sur Habrobracon juglandis Ashmead, Braconide célèbre par les nombreuses recherches de génétique dont il fut l'objet. Ses cufs ont èté étudiés par H. MaERcks (1933). Ils sont largement euryhygres, leur optimum vital ( $\left.{ }^{1}\right)$ est à $80 \%$ d'humidité relative et ils peuvent encore donner des éclosions à $4 \%$ d'humidité relative. Leur développement n'est guère influencé : aux températures optimales, la vitesse du développement est la même à tous les taux d'humidité ; aux températures suboptimales ot superoptimales, la sécheresse allonge notoirement, le voisinage de la saturation allonge faiblement la durée du développement. E. Hoppe (1937) a étuaié la longévité des adultes de deux souches du Habrobracon juglandis. Pour les deux cas, les vies les plus longues ont èté notées à 50-60\% d'humidité relative. En atmosphère saturée, les sujets vivent moins longtemps,à $12 \%$ d'humidité relative moins encore.

N. Payne (1933) a publié les résultats de ses recherches sur le Microbracon hebetor Say, Braconide parasite d'Ephestia kuehniella Zeller (Lep.). Les œufs n'en seraient influencés par les conditions d'humidité, ni dans leur vitalité, ni dans leur développement. Les larves sont faiblement euryhygres et défavorisées par une trop grande sécheresse. Les nymphes, qui vivent à l'air libre, sont au contraire extrêmement résistantes à la dessiccation.

Pour clore cet exposé bibliographique, rapportons que P. Voukassovitch (1929) a montré que les cocons d'un autre Braconide : Macrocentrus abdominalis $F$. ne peuvent être bien formés en atmosphère saturée d'humidité. Par ailleurs, les nymphes résistent bien à la sécheresse et donnent, en moyenne, leurs éclosions plus vite en milieu sec. Les adultes des deux sexes du même Macrocentrus vivent beaucoup plus de jours en milieu sec qu'en milieu relativement humide. Cette espèce peut donc être considérée comme nettement hygrophobe.

\section{EXPÉRIMENTATION PERSONNELLE.}

J'ai étudié l'influence de l'humidité sur la nymphose de l'A panteles glomeratus L., parasite abondant en nos régions $\left(^{(}\right)$des chenilles de Pieris rapae L. (1). L'humidité

(1) J'utilise cette expression pour désigner les conditions hygrométriques qui, dans les thermo: hygrogrammes des écologistes allemands, correspondent aux mortalités minimalés pour le plus grand nombre des températures vitales.

(2) Je dois la détermination de ce parasite à M. H. De Sargek (Bruxelles). 
ne semble guère avoir d'effels sur le développement larvaire, lukAYA (1938) faiisait a'ailleurs la même remarque pour un Apanteles japonais : l'A panteles militaris. Il n'en est plus de même lorsque la larve a terminé sa vie d'endoparasite. Il faut dire en effet que chez beaucoup de Braconides (Apanteles, Macrocentrus) les larves accomplissent leur croissance à l'intéricur des tissus de l'hôte, puis deviennent parasites externes quelques heures avant leur nymphose. Ce stade critique où se produisent de profonds changements physiologiques est très résistant à la sécheresse : j'ai obtenu des sorties de larves et leur transformation même à $0 \%$ d'humidité relative (température du laboratoire en été). Par contre, la saturation est très mal supportée : les larves y lardent à se dégager des téguments de leur victime, elles n'arrivent presque jamais à tisser leur cocon et à fortiori à nymphoser ; dans ces conditions elles meurent souvent après deux ou trois jours d'efforts prolongés. Les cocons, aussitôt tissés en milieu plus favorable, ont perdu cette sensibilité à l'excès hygrométrique, ils manifestent une différence totale aux conditions d'humidité tant pour la durée de leur développement que pour leur vilalité. L'A panteles glomeratus parait donc réagir à l'humidité de la même façon que le Macrocentrus abdominalis étudié par P. Voukassovitch (1929).

\section{Hyménoptères Aculéates}

(fouisseurs)

On ne trouve dans la littéra'ure aucun renseignement sur la sensibilité à l'humilité des larves et des nymphes d'Hyménoptères fouisseurs. J'ai pu soumettre à l'expérience deux espèces de rubicoles de la famille des Sphégides : Passalocus gracilis Curtis el Crabro rubicola D. et P.

Le Passalocus gracilis Curtis établit ses cellules dans les tiges creuses de ronce, sureau, fusain, etc. J'ai publié antérieurement des détails sur la nidification de celle espèce prédatrice de pucerons (J. Leclence, 1939, 1940, 1941). En mars 1943 , j'ouvrais une série de tiges habilées par des prénymphes de Passalocus et j'exposais ces larves à $100 \%$ el à $55 \%$ d'humidité relative (température extérieure $\pm 13^{\circ}$ C.). En moins de 24 heures, les larves placées à $55 \%$ étaicnt mortes, séchées et recroquevillées. Celles placées à $100 \%$ ne subirent aucun dommage et donnèrent normalement nymphes et adultes fin-avril.

Le Crabro rubicola D. et P. (larvalus Wesmael) établit ses cellules dans les iiges creuses de sureau (J. Leclerce, 1941). On sait que les larves de Crabro s'entourent, pendant leur croissance d'un cocon brun, de nature inconnue. J'ai exposé quelquesuns de ces cocons à $100,85,75$ et $55 \%$ d'humidité relative, au début de mars, c'est-à-dire à un moment où les cocons renferment une prénymphe on hibernation, Nymphoses et éclosions se produisirent normalement partout à la fin avril et, aux conditions de lempéralure utilisées $\left(14\right.$ à $19^{\circ} \mathrm{C}$.), je n'ai noté aucun échec.

Ces deux résultats paraissent indiquer que les larves hivernantes des Sphégides rubicoles ne sont guère protégées contre la dessiccation, sauf quand elles sont entourées d'un cocon.

\section{EXPÉRIENGES AVEG LES FourMis}

K. Gösswald (1938) a consacré tout un mémoire à l'étude de la résistance des fourmis au jeûne, en fonction de la température et de l'humidilé. Toutesles espèces 
qu'il étudia, qu'elles soient hygrophiles ou xérophiles dans la nalure, lrouvent à $100 \%$ d'humidité relative les conditions optimales pour la longévité des ouvrières. Toutes les fourmis se comportent comme "sténohygres "lorsqu'on les soumel au jeûne.Toutefois on peul constater des différences en rapport avec le mode de vie et les biotopes habituels des espèces, ainsi Lasius niger et Telramorium caespitum qui sont hygrophiles et nidifient en terre sont plus sensibles à la sécheresse que les Leptothorax qui sont xérophiles et ont des nids plus aériens.

K. Gösswald (1941) a consacré un second mémoire à l'étude du sens de l'humidité (Luftfeuchtigkeitssinn) des fourmis. Toutes les espèces étudièes réagissent dans les hygrocinomètres (Luf!feuchtigkeitsorgel) en choisissant l'almosphère sitturée de préférence à tous les autres taux. Les fourmis réagissent avec d'aulant plus de célérité et d'autant plus de constance qu'elles appartiennent à des espèces écologiquement plus hygrophiles.

J'ai vérifié cette prédilection des fourmis pour l'atmosphère humide dans lè cas particulier des ouvrières de Lasius niger L. Mes essais ont élé réalisés à l'aide d'un hygrocinomètre comparable à celui de K. GosswaLd (194l) el présentant aux sujets expérimentaux huit condilions hygrométriques comprises entre 10 et $100 \%$ d'humidité. Tous les essais mirent en évidence le même hygrotropisme. J'ai réussi au surplus l'expérience suivante qui ajoute un fait original aux données de K. GössW:ALD :

Le 24 août 1943, j'introduis, dans le compartiment le plus sec de mon hygrocinomètre, une trentaine d'ouvrières de Lasius niger et une cinquantaine d'œufs, larves et nymphes. Température aurant l'expérience : $23^{\circ} \mathrm{C}$.

Le premier travail des fourmis fut d'entasser. en hâte la progénilure contre la paroi la plus proche. Quelques ouvrières cependant firent le tour de tout l'appareil (lequel avait $50 \mathrm{~cm}$. de long et était divisé par sept cloisons disposées en chicane) et une dizaine d'entre elles se trouvaient, après quelques heures, réunies à $100 \%$ d'humidité relative. Le lendemain matin, lout ce que j'avais introduit dans le compartiment sec était cantonné dans le compartiment saturé, les ouvrières ayạnt transporté œufs, larves et nymphes à l'endroit où les conditions hygrométriques optimales étaient réalisées.

\section{Conclusions}

1. - La plupart des formes d'Hyménopières étudiées jusqu'ici se sont révélées sensibles à l'humidité atmosphérique. Les formes parasites sont toujours euryhygres, il est probable que les formes phytophages le sont aussi généralement. Certains stades, protégés par le cocon d'un hôte ou par un cocon individuel ne subissent évidemment guère les effets de l'humidité relalive extérieure. Les fourmis, au contraire, sont neltement sténohygres de même que les larves de Passaloecus. ll y a, comme on peut le voir, une relation certaine entre le mode de vie spécifique et la réaction à l'humidité.

2. - Les stades prénymphaux ectoparasites de certains Térébrants (Macrocentrus, Apanteles) ne peuvent supporter la saturation hygrométrique.

3. - Les fourmis sont capables de choisir, dans un gradient hygrométrique, les conditions qui leur sont optimales. 


\section{Sources Bibliographiques}

Ashber, R. (1932). - La Respirazione e l'Influenza della Temperatura e dell'Umidita sulle Larve dell'Eurytoma amygdali End. (Arch. Sc. Biol., 17, 293).

Fukaya (1938). - Cité par H. De SaEger : Les Apanteles, Hyménoptères Bracenides, parasites de Lépidoptères (Bull. Agric. Congo Belge, 33, 1942, 234).

Geisthardt, G. (1937). - Ueber die ökologische Valenz zweier Wanzenarten mit verschiedenen Verbreitungsgebiet $(Z$. Parasitenkunde, 9, 151).

GiARD, A. (1894). - L'anhydrobiose ou ralentissement des phénomènes vitaux sous l'influence de la déshydratation progressive $(G$. R. Séances Soc. Biol., 16, 497).

Gösswald, K. (1935). - Physiologische Untersuchungen über die Einwirkung ökologischer Faktoren, besonders Temper. und Luftfeucht., auf die Entwicklung von Diprion (Lophyrus) pini L... ( $Z$. angewandte Entom., 22, 331).

Gösswald, K. (1938). - Ueber den Einfluss von verschiedenen Temperatur und Luftfeuchtigkeit auf die Lebensäusserungen der Ameisen. I ( $Z$. wissenschafliche Zool., 151, 337.)

Gösswald, K. (1941). - Id. II (Z. wissenschaflliche Zool., 154, 247.)

HopPE, E. (1937). - Untersuchungen über die Vitalität zweier Stämme von $H a-$ brobracon juglandis Ashmead und verschiedenen Bedingungen $(Z$. angewandte Entom., 23, 559).

Leclerce, J. (1939). - La Biologie des Passaloecus (Lambillionea, 39, 59.)

LeClercQ, J. (1940). - Id. (2e.note). (Lambillionea, 40, 49.)

LechercQ, J. (1941). - Notes sur les Hyménoptères des environs de Liége (Bull. Mus. roy. Hist. nalur. Belg., 17, no 14.)

Lund, H. O. (1934). - Some Temperature and Humidity Relations of two Races of Trichogramma minutum Riley (Ann. Entom. Soc. America, 27, 324).

MaEncks, H. (1933). - Der Einfluss von Temperatur und Luftfeuchtigkeit auf die Embryonalentwicklung der Mehlmottenschlupfwespe, Habrobracon juglandis Ashmead (Arb. biol. Reichsanstalt Land-und Forstwirtschaft, 20, 347).

PAyne, N. M. (1933). - The differential Effect of environmental Factors upon Microbracon hebetor Say and its Host Ephestia kuehniella Zeller (Biol. Btill., 65, 187.)

Ullyett, G. G. (1936). - Physical Ecology of Microplectron fuscipennis (Bull. Entom. Res., 27, 195).

Voukassovitch P. (1929). - Contribution à l'étude de Macrocentrus abdominalis F. et de ses parasites (Ann. Soc. ent. Fr., 98, 163.)

Le Secrétaire-gérant: L. Chopand. 